\title{
EXPERIMENTAL AND NUMERICAL STUDY OF THE ROTATING DETONATION ENGINE IN HYDROGEN-AIR MIXTURES
}

\author{
J. Kindracki ${ }^{1}$, A. Kobiera ${ }^{1}$, P. Wolański ${ }^{1}$, Z. Gut ${ }^{1}$, \\ M. Folusiak ${ }^{2}$, and K. Świderski ${ }^{2}$ \\ ${ }^{1}$ Warsaw University of Technology \\ ul. Nowowiejska 21/25, Warsaw 00-665, Poland \\ ${ }^{2}$ Institute of Aviation \\ al. Krakowska 110/114, Warsaw 02-256, Poland
}

\begin{abstract}
Experimental and numerical study of rotating detonation is presented. The experimental study is focused on the evaluation of the geometry of the detonation chamber and the conditions at which the rotating detonation can propagate in cylindrical channels. Lean hydrogen-air mixtures were tested in the experiments. The pressure measured at different locations was used to check the detonative nature of combustion. Also, the relationship between detonation velocity and operation conditions is analyzed in the paper. The experimental study is accompanied with numerical analysis. The paper briefly presents the results of two-dimensional (2D) numerical simulation of detonative combustion. The detonating mixture is created by mixting hydrogen with air. The air is injected axially to the chamber and hydrogen is injected through the inner wall of the chamber in radial direction. Application of proper injection conditions (pressure and nozzle area) allows establishing a stable rotating detonation like in the experiments. The detonation can be sustained for some range of conditions which are studied herein. The analysis of mean parameters of the process is provided as well. The numerical simulation results agree well with the experiments.
\end{abstract}

\section{INTRODUCTION}

Modern classical jet and rocket engines have almost reached a level when it is very difficult to increase their performances by minor modification or improving material parameters. In order to achieve a significant increase in thermal efficiency of such an engine, some extraordinary means must be applied, e.g., changing the thermodynamic cycle of the engine. This goal can be achieved by

This is an Open Access article distributed under the terms of the Creative Commons Attribution-Noncommercial License 3.0, which permits unrestricted use, distribution, and reproduction in any noncommercial medium, provided the original work is properly cited. 
using the detonation mode of combustion instead of deflagration. Detonative combustion can be realized in different ways, for example: by application of pulsed detonation process in so-called Pulsed Detonation Engine (PDE), or continuously rotating detonation in so-called Rotating Detonation Engine (RDE) (also known as Continuous Detonation Wave Engine). Advantages of PDE are: relatively simple design, and high efficiency. The disadvantages are: low frequency, necessity of initiation of each cycle, large length and initiation distance, time variation of thrust, high level of noise, and intense vibrations. Contrary to $\mathrm{PDE}$, in $\mathrm{RDE}$, the detonation once initiated propagates continuously in a cylindrical chamber, the engine is more compact and efficient, and smaller variations of thrust are created if any. In addition, the RDE provides a possibility of reducing NOx emission due to the use of lean mixtures which are burnt at low temperature compared to that obtained in stoichiometric region of combustion in classical burners.

Recently, the interest of detonation researchers has been focused on the development of the RDE since it offers significant improvements in thermal efficiency and simplifications in design. Many experimental studies have been conducted in this field at numerous laboratories. In early 1960s, Voitsekhovskii, Mitrofanov, and Topchiyan performed first experiments on continuously rotating detonation $[2,1]$. In 2004, Wolanski and Fujiwara in cooperation with Mitsubishi Co. applied for a patent on the RDE [3]. The principle of RDE operation is based on generation of a continuously propagating detonation in a disk-like combustion chamber (toroidal or ring-like shape). The fresh mixture is supplied from one side and combustion products are escaping from the other side of the chamber.

Research on RDE is carried out in Russia, France, Poland, Singapore, China, and Japan. Many important publications on this topic have been published for last five years. Bykovskii et al. [4-7] reported the results of experimental studies of the continuously rotating detonation. Hishida et al. [8] presented theoretical backgrounds and numerical calculation. Davidenko et al. [9, 10] reported the results of numerical simulations of rotating detonation in a rocket engine. $\mathrm{Yi}$ et al. presented a comparison of PDE and RDE [11]. Bykovskii et al. [7] described the results of experimental study for hydrogen-oxygen mixture. Very extensive experimental and numerical studies on the application of rotating detonation to propulsion systems are carried out in Poland [12-14].

\section{EXPERIMENTAL SETUP}

The general layout of the experiment is shown in Fig. 1. The air is injected in axial direction to the detonation chamber through the slit with the cross-section area smaller than that of the chamber. Fuel injectors are placed at the end of the 


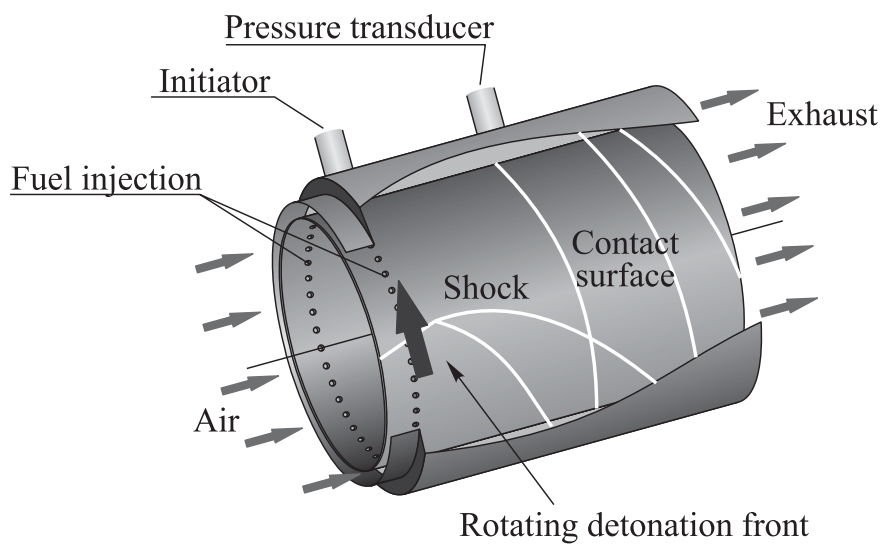

Figure 1 Sketch of detonation chamber used in the experiments

slit. The injection nozzles are located at the inner surface of the chamber. This ensures proper mixing. The detonable mixture is created downstream of the injectors. After initiation, the rotating detonation propagates in the chamber. Owing to efficient mixing, the detonation is capable of moving very close to the injectors as is shown in Fig. 1. The detonation front generates secondary shock waves which spread upstream and downstream.

The experimental setup consists of the following parts (Fig 2): detonation chamber 1, damp tank 3, fuel and air feed system, measurement system, and initiation system. Since the main part of the study dealt with seeking the optimal geometry for the detonation, the cylindrical detonation chamber (see Fig. 1) has been built with the use of easily replaceable elements. It allows changing internal diameter of the chamber, length of the channel, and shape of the chamber inner wall. The chamber was equipped with several pressure transducers. Two of them were placed in the manifolds and the others were installed into the chamber at locations shown in Fig. 2. The detonation chamber was connected to the dump tank (volume about $0.63 \mathrm{~m}^{3}$ ). Therefore, it was possible to conduct experiments in the wide range of initial pressure $(0.05-2.5$ bar) by controlling pressure in the dump tank. The view of the setup is also shown in Fig. 2.

Fuel and air were injected to the chamber by two ways: fuel through the large number of small holes $(0.7-1.0 \mathrm{~mm}$ in diameter) and air through a narrow slit of variable width $(0.5-2 \mathrm{~mm})$. Fuel and air injectors into the cylindrical chamber were controlled by two electromagnetic valves operated automatically by an electronic control unit.

The rotating detonation was initiated by a strong shock wave produced by a gaseous initiator attached to the detonation chamber. The initiator was filled with high-pressure stiochiometric acetylene-oxygen mixture and ignited by an electric spark. A thin plastic diaphragm was placed between the chamber and 


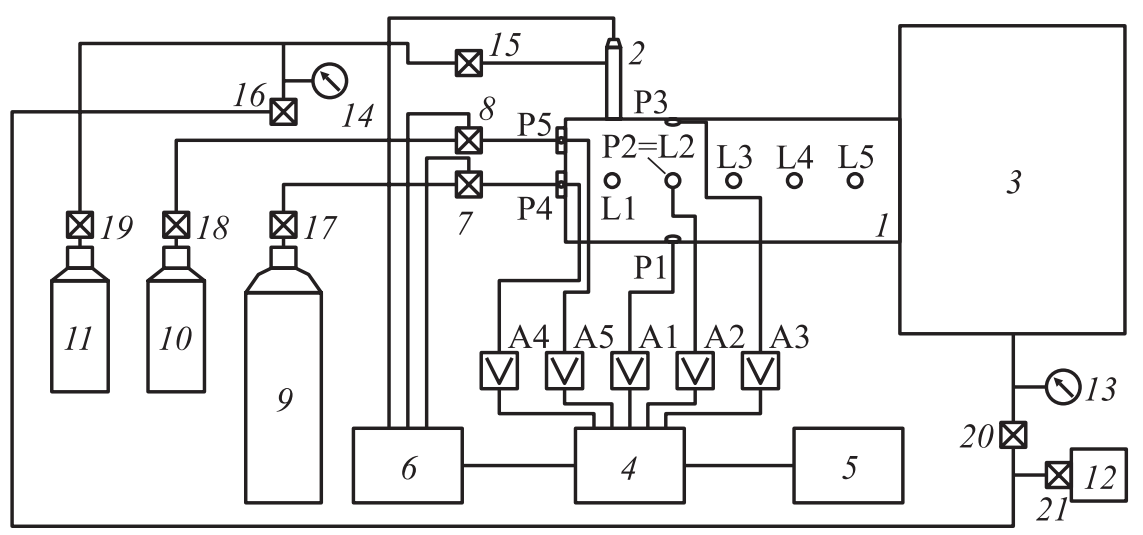

(a)

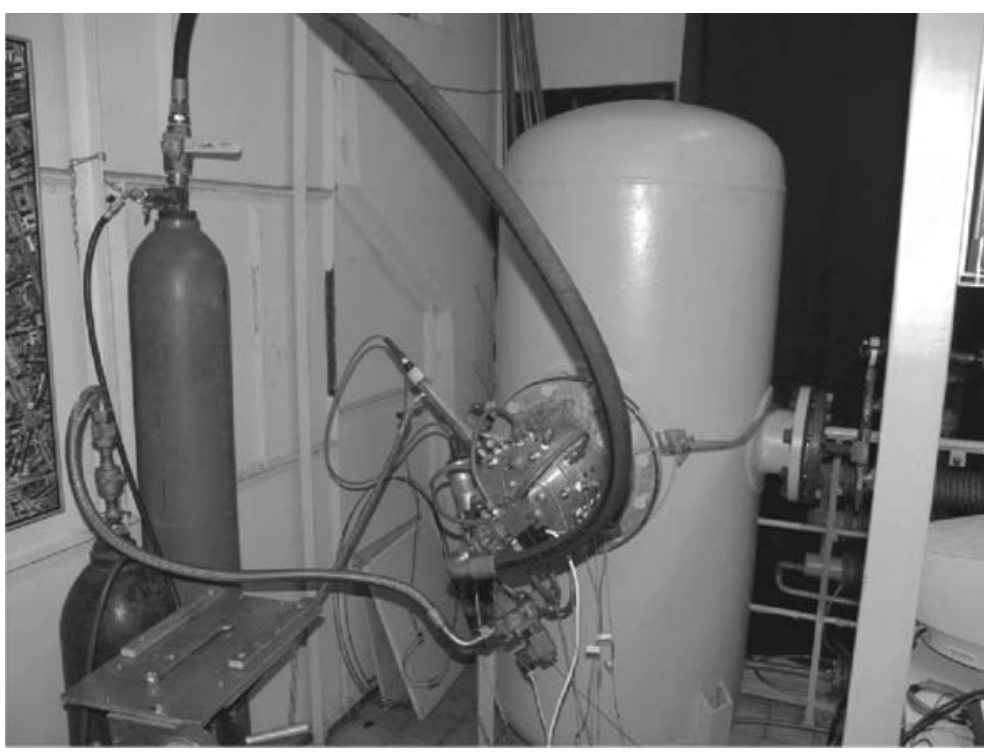

(b)

Figure 2 Small experimental rig: (a) schematic diagram: P1-P3 - pressure transducers placed in one plane inside the chamber; L1-L5 - pressure transducers placed in one line inside the chamber; P4, P5 - pressure transducers for manifolds: fuel and air; A1-A5 - amplifiers; 1 - detonation chamber; 2 - initiation tube; 3 - dump tank; 4 - acquisition card; 5 - computer; 6 - control system, 7 and 8 - electromagnetic valves; 9 - tank with air; 10 - tank with fuel; 11 - bottle with initiation mixture; 12 - vacuum pump; 13 and 14 - manometers; and 15-21 - valves; and (b) view of the stand 


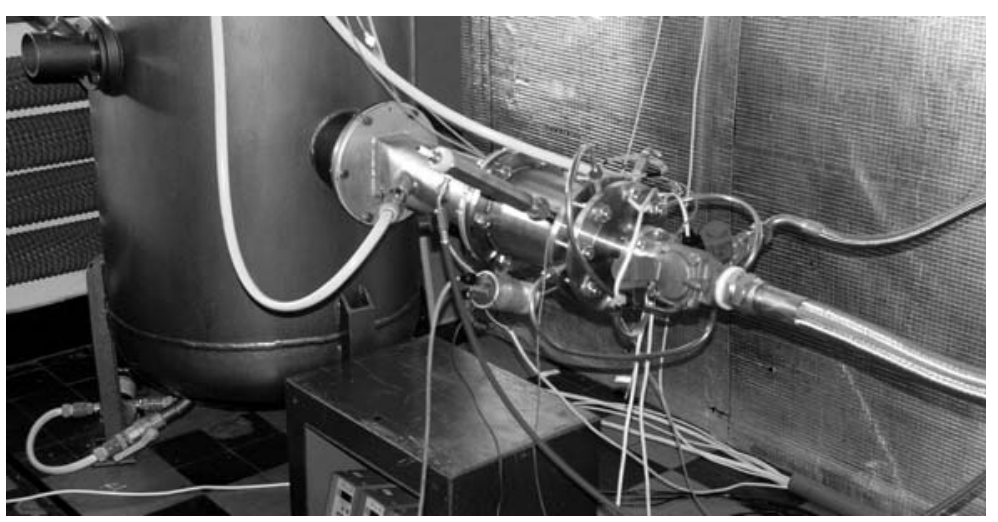

Figure 3 Experimental rig for continuous flow operation

the initiator for separation these volumes before experiments. Immediately after fuel and air injections, the electric spark was used to detonate the mixture in the initiator. It produced a strong shock wave in the main chamber, which eventually triggered the rotating detonation.

This general setup was applied in two test rigs. The first was smaller and was used for short-duration experiments (Fig. 2 b). The second was larger and was used for longer test duration (Fig. 3). Typical short experimental time was 80-150 ms but in some cases, it was extended up to $1 \mathrm{~s}$. The time depended on the initial pressure in the chamber or on the cooling of pressure transducers.

The rig for longer, continuous operations was equipped with bigger air and fuel tanks. The dump tank was connected to the atmosphere, so the experiments could be run up to $5 \mathrm{~s}$. The limitation of experiment duration was the capacity of the fuel and air tanks. Anyway, the time was long enough to show the possibility of continuous chamber operation with the rotating detonation. Additionally, exhaust gas temperature was measured by a thermocouple with relatively small response time. For pressure measurements, Kistler pressure gauges were used. To protect the gauges from intense heating by hot detonation products, they were covered by a small layer of silicon grease.

\section{EXPERIMENTAL RESULTS}

The experiments for hydrogen-air mixture were carried out for three geometries:

(i) small chamber (outer diameter $95 \mathrm{~mm}$ );

(ii) medium chamber (outer diameter $150 \mathrm{~mm}$ ); and

(iii) continuous-flow medium chamber (outer diameter $134 \mathrm{~mm}$ ). 


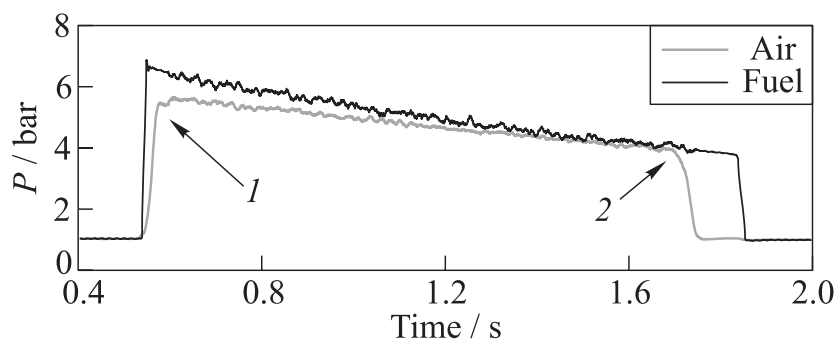

Figure 4 Typical pressure records in the manifolds for fuel and oxidizer during experiments: 1 - detonation initiation in the chamber; and 2 - closing of feed valves

In all cases, the pressure was measured in the manifolds and in the chamber. The detonation velocity and flow rates of both gases were calculated from pressure records. Additionally, for long duration experiments (larger test rig), exhaust gas temperature was also measured.

Figure 4 shows the pressure histories in the manifolds; point 1 is the initiation time, and point 2 indicates closing of the valves which cut off the flow from the air and fuel tanks (for continuous flow operation). The observed pressure drop in the manifolds is explained by the small volume of tanks with fuel and air. For the case presented in Fig. 4, the volume of the fuel tank was only $69 \mathrm{l}$ which ensured only $5-6 \mathrm{~s}$ of continuous operation. Nevertheless, even for such a short time, it was possible to measure all mentioned parameters and to optimize the detonation chamber geometry.

\subsection{Velocity and Temperature Measurements}

The instantaneous detonation velocity, $V_{\text {inst }}$, was calculated from the period $\Delta t$ between successive pressure peaks measured during the experiment, namely, $V_{\text {inst }}=\pi D_{\mathrm{ch}} / \Delta t$ (Fig. 5) where $D_{\mathrm{ch}}$ is the diameter of the chamber. Since the calculations were made for all detonation peaks, different values of detonation velocity were obtained. Therefore, the detonation velocity was the mean value of $V_{\text {inst. }}$. The results will be discussed later.

Figure 6 shows the temperature measurements by thermocouple. A typical temperature profile obtained during experiments can be divided into three parts: $T_{n}$ is the time period required for the stabilization of measurement (which depends on the thermocouple response time). After this time, the temperature reaches a steady level. These measurements indicate the real temperature of the products, which depends mainly on mixture composition and pressure in the chamber. After closing fuel and air supply valves, the temperature decreases mainly due to stopping exhaust gases and cooling the thermocouple. 


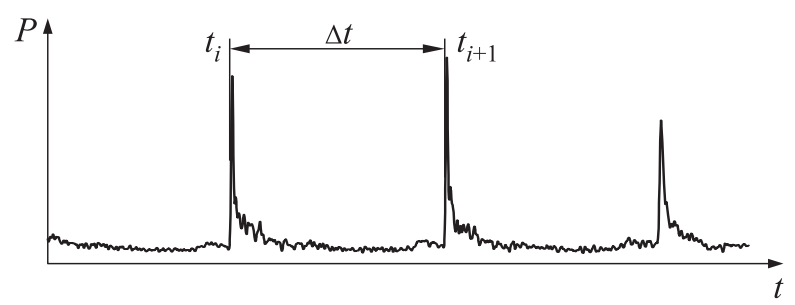

Figure 5 Detonation velocity measurements with pressure records

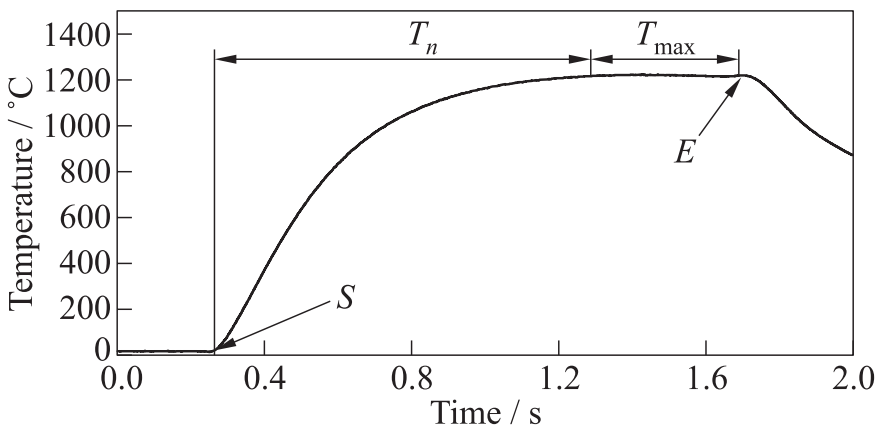

Figure 6 Layout of exhaust gas temperature measurements ( $S$ is the instant of detonation initiation; $E$ is the time of closing the gas supply system)

\subsection{Pressure Measurements}

The pressure gauges are subject to extreme conditions of detonation and should measure pressure variation with high frequency and high accuracy. The main problem is high-temperature environment. In order to counteract overheating of the gauge, the front face of the gauge was covered by a layer of the silicon grease. However, the thickness of the layer influenced the measurements.

Figures $7 a$ to $9 a$ show three cases of measurements for the same hydrogenair mixture with different thickness of the silicon grease: 3,1 , and $0.3 \mathrm{~mm}$, respectively. This comparison provides useful information on the distortion of pressure signal by a silicon grease layer. It is noticeable, especially for the thicker silicon layer, that the layer influences on the shape of pressure profile, peak pressure (usually, lower pressure is indicated), and has some effect on the calculated detonation velocity.

Figures $7 b$ to $9 b$ show that for the 3-millimeter thick layer, the calculated velocity is about $1400 \mathrm{~m} / \mathrm{s}$ with $\pm 300 \mathrm{~m} / \mathrm{s}$ oscillations (for period $0.4 \mathrm{~s}$ ), while for 0.3 -millimeter layer, this value is $1500 \pm 50 \mathrm{~m} / \mathrm{s}$. Concluding, very small grease 


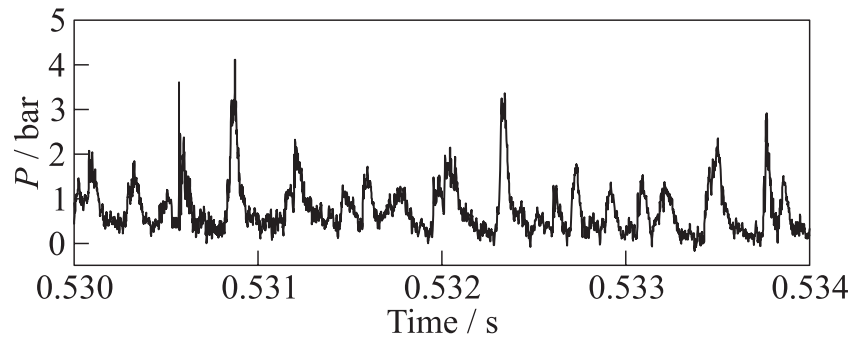

(a)

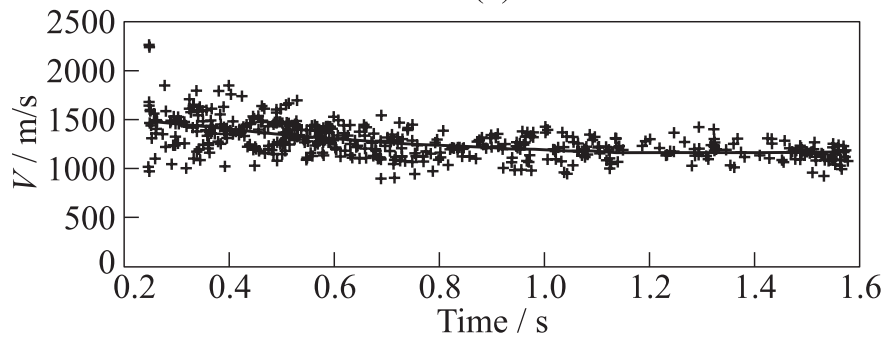

(b)

Figure 7 Experimental measurements for pressure gauge with 3-millimeter thick silicon grease layer: $(a)$ pressure-time history; and $(b)$ calculated detonation velocity

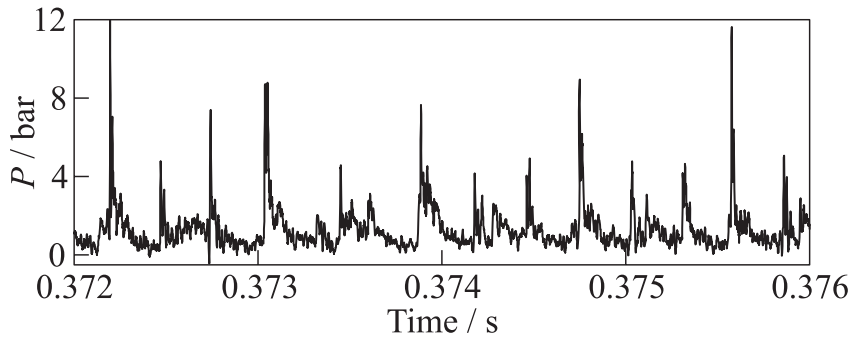

(a)

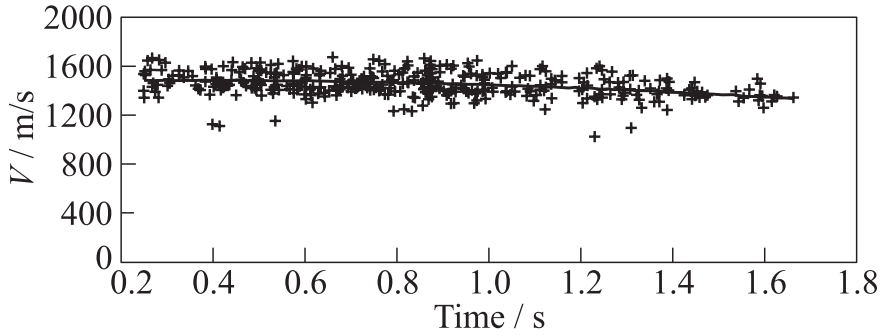

(b)

Figure 8 Experimental measurements for pressure gauge with 1-millimeter thick silicon grease layer: $(a)$ pressure-time history; and (b) calculated detonation velocity 


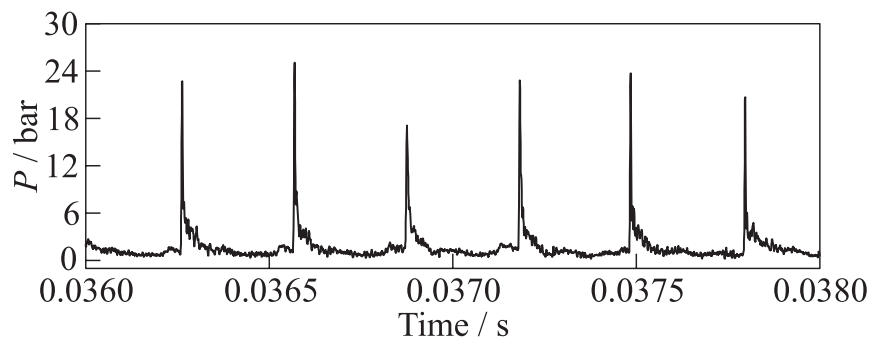

(a)

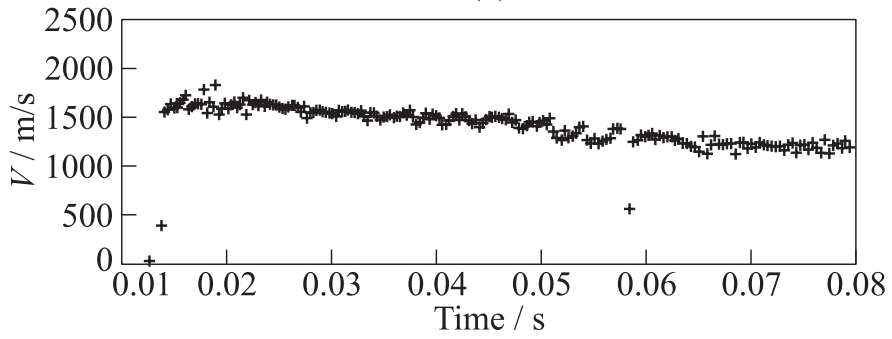

(b)

Figure 9 Experimental measurements for pressure gauge with 0.3-millimeter thick silicon grease layer: $(a)$ pressure-time history; and (b) detonation velocity

layer and short duration experiments are necessary for examining the structure of the detonation front, but for the temperature measurements of the exhaust gas, the experimental time must be extended and a thick layer for protecting the pressure transducer surface must be used.

\subsection{Influence of Chamber Diameter and Channel Width}

Experiments were carried out in chambers with three different values of outer diameter. In each case, it was possible to change the width of the channel from 5 to $20 \mathrm{~mm}$. Figures 10 and 11 show pressure records and detonation velocities as functions of time for two different channel widths. In both experiments, the initial pressure in the chamber was 1 bar. Clearly, the pressure peaks were not high and the detonation was unstable. For the small channel, the time of one revolution of the detonation front was relatively short; consequently, the refilling time was short and the fuel and air did not sufficiently mix. If the fuel and air are not properly mixed, the detonation parameters decrease (the detonation pressure and detonation velocity become lower) and the time of detonation revolution increases. This improves the quality of mixing for the next cycle and thus increases the observed detonation pressure and velocity. This constitutes 


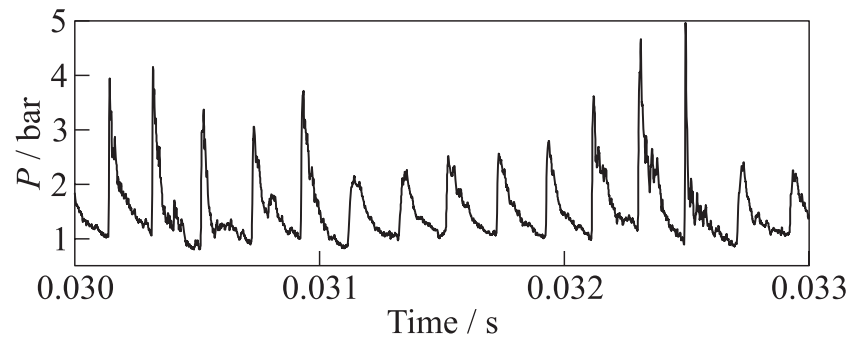

(a)

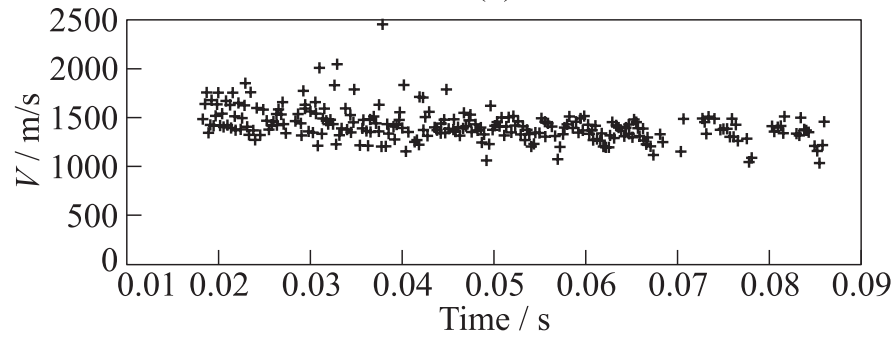

(b)

Figure 10 Detonation in hydrogen-air mixture in 95-millimeter chamber (5-millimeter channel width): (a) pressure record; and (b) detonation velocity

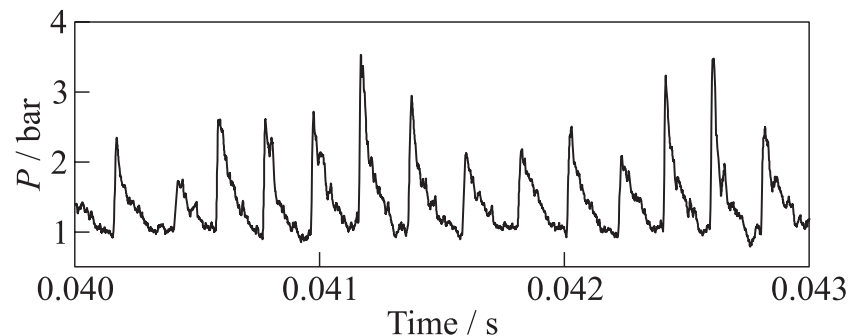

(a)

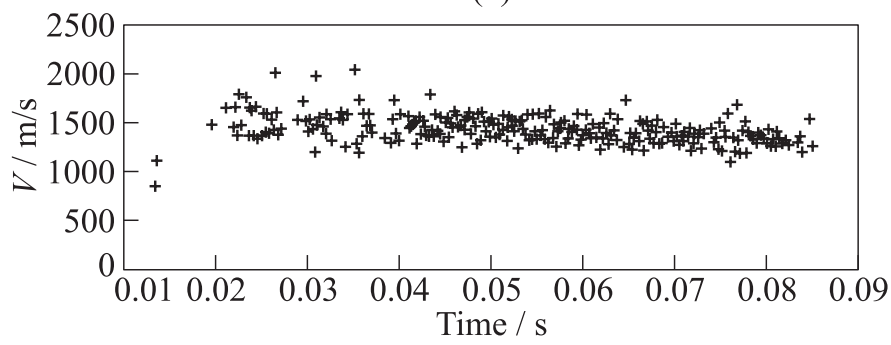

(b)

Figure 11 Detonation in hydrogen-air mixture in 95-millimeter chamber (10millimeter channel width): (a) pressure record; and $(b)$ detonation velocity 


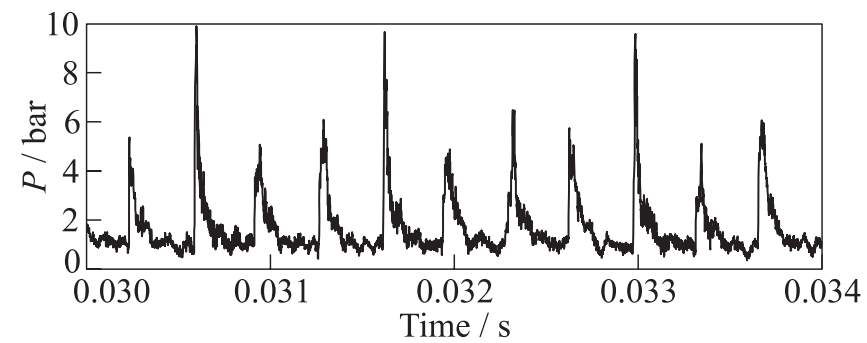

(a)

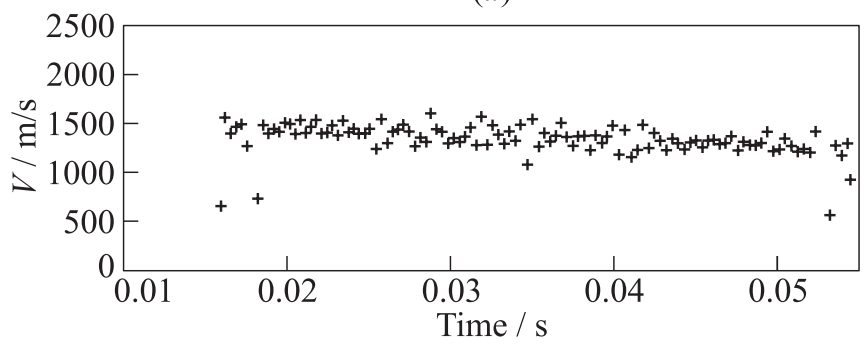

(b)

Figure 12 Detonation in hydrogen-air mixture in 150-millimeter chamber (5-millimeter channel width): (a) pressure records; and (b) detonation velocity

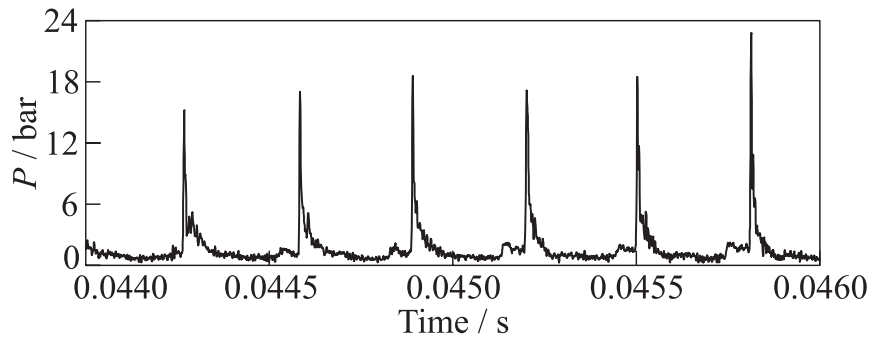

(a)

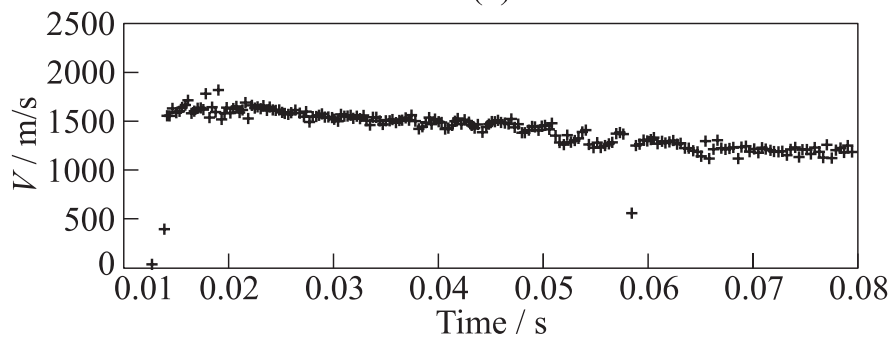

(b)

Figure 13 Detonation in hydrogen-air mixture in 150-millimeter chamber (10millimeter channel width): (a) pressure records; and (b) detonation velocity 
the instability mechanism which leads to observed pressure and velocity fluctuations. In such cases, the pressure fluctuations are high and the detonation velocity varies approximately from 1000 to $2000 \mathrm{~m} / \mathrm{s}$.

Figures 12 and 13 present the results for the chamber of 150-millimeter diameter. Two channel widths were used: 5 and $10 \mathrm{~mm}$. One can notice that 10-millimeter channel gives better results, with nearly the same pressure peaks, and very stable detonation. The deviation of the velocity around the mean value is less than $4 \%$. In this case, there is sufficient time for mixture formation during one revolution of the detonation wave. The revolution time is nearly a factor of 2.5 longer than in the small chamber for the same mixing parameters. It means that selection of the proper geometry is the crucial for eliminating the instability. When the diameter of the detonation chamber increases to a larger size, the mixing time might be too long and two or more detonation waves could propagate in the chamber.

\subsection{Temperature as a Function of Equivalence Ratio}

Many experiments were carried out for temperature measurements. Figure 14 shows the results as a function of mixture equivalence ratio $\Phi$. For average equivalence ratio in the range of $0.25-0.6$, the measured temperature was between 800 and $1200{ }^{\circ} \mathrm{C}$. It should be also noted that the realistic value of $\Phi$ in the detonation region might be higher due to imperfect mixing of hydrogen with air. The measured values of temperature are in good agreement with the results of numerical simulations presented in the next part of this paper. It demonstrates the possibility of using the chamber with rotating detonations as a combustion chamber in a conventional jet engine. The low temperature at the exit is achieved here without air dilution. This can significantly simplify the geometry of the combustion chamber reducing its size and weight. This solution can also improve thermal efficiency [14].

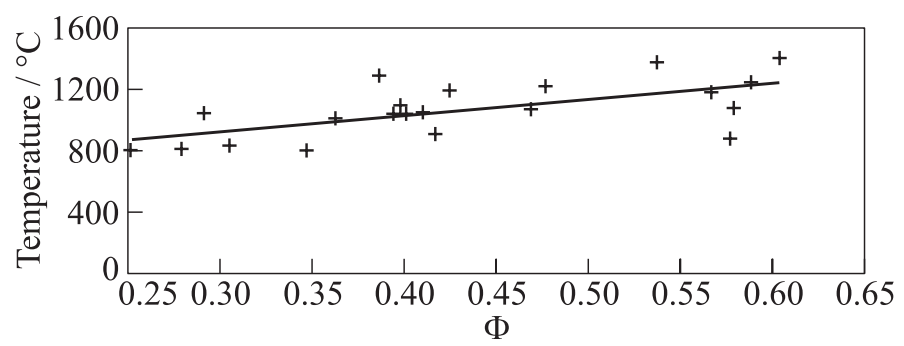

Figure 14 Measured temperature of exhaust gas as a function of hydrogen-air mixture equivalence ratio 


\section{NUMERICAL SIMULATION MODEL}

The 2D simulations of the rotating detonation have been already conducted by Davidenko et al. [10], Yi et al. [15], and other researchers. However, they simulated a detonation propagating in a premixed gas mixture. In the present calculations, it is assumed that the detonation chamber is fed with fresh air and hydrogen which is injected at some distance from the inflow boundary.

Figure 15 shows the scheme of the detonation chamber and the computational domain. The injection is assumed to be normal to the plane of the computational domain. It is simulated as a source term in the equations of total and partial densities as well as in the equation of energy conservation. This approach means that the mixing of fuel and air is instantaneous in the chamber.

The simulations are based on the classical Euler equations describing the motion of a reacting inviscid gas:

$$
\frac{\partial Q}{\partial t}=-\frac{\partial E}{\partial x}-\frac{\partial F}{\partial y}+S+S_{\mathrm{inj}}
$$

where

$$
Q=\left(\begin{array}{c}
\rho \\
\rho u \\
\rho v \\
e \\
\rho_{i}
\end{array}\right) ; \quad E=\left(\begin{array}{c}
\rho u \\
\rho u^{2}+p \\
\rho v u \\
(e+p) u \\
\rho_{i} u
\end{array}\right) ; \quad F=\left(\begin{array}{c}
\rho v \\
\rho u v \\
\rho v^{2}+p \\
(e+p) v \\
\rho_{i} v
\end{array}\right)
$$

(a)

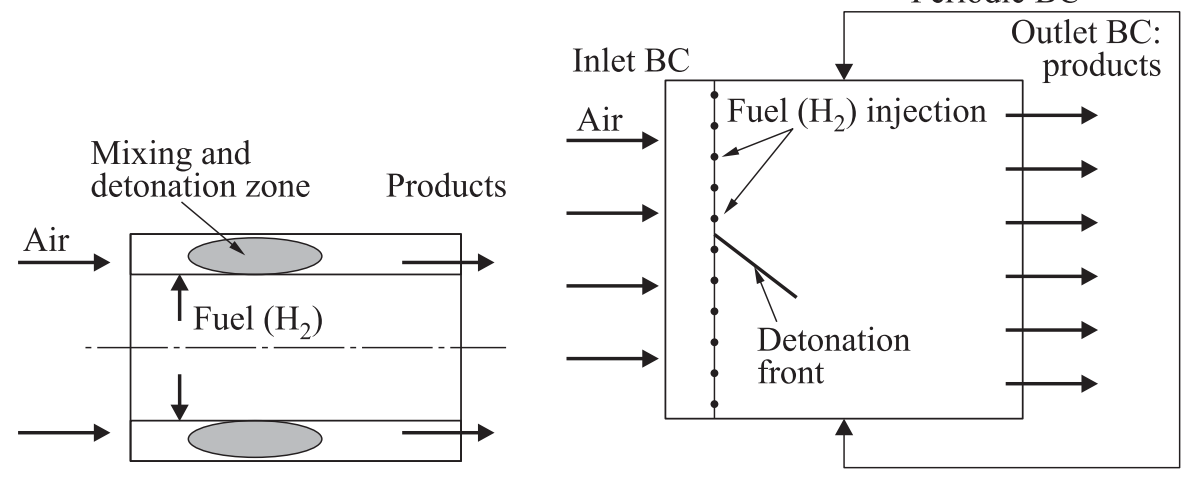

Periodic BC

(b)

Figure 15 Simplified scheme of detonation chamber $(a)$ and the computational domain with boundary conditions $(b)$ 


$$
S=\left(\begin{array}{c}
0 \\
0 \\
0 \\
0 \\
\omega_{i}
\end{array}\right) ; \quad S_{\mathrm{inj}}=\left(\begin{array}{c}
\dot{\rho}_{\mathrm{inj}} \\
0 \\
0 \\
\dot{\rho}_{\mathrm{inj}} h_{\mathrm{inj}} \\
\dot{\rho}_{\mathrm{inj} i}
\end{array}\right) .
$$

Injection source term $S_{\text {inj }}$ is a function of injection pressure $p_{\text {inj }}$, temperature $T_{\text {inj }}$, the ratio of nozzle area to the wall area $f_{\text {nozzle, }}$ and height of the channel $h_{\mathrm{ch}}$ :

- subsonic flow

$$
\dot{\rho}_{\text {inj }}=\frac{p_{\text {inj }}}{\sqrt{R T_{\text {inj }}}} \sqrt{\frac{2 \gamma}{\gamma-1}\left[\left(\frac{p}{p_{\text {inj }}}\right)^{2 / \gamma}-\left(\frac{p}{p_{\text {inj }}}\right)^{(\gamma-1) / \gamma}\right]} \frac{f_{\text {nozzle }}}{h_{\text {ch }}} ;
$$

- for sonic flow

$$
\dot{\rho}_{\text {inj }}=\frac{p_{\text {inj }}}{\sqrt{R T_{\text {inj }}}} \sqrt{\gamma\left(\frac{2}{\gamma+1}\right)^{(\gamma+1) /(\gamma-1)}} \frac{f_{\text {nozzle }}}{h_{\text {ch }}} .
$$

The source term in the energy equation is a product of the density source term and total enthalpy of the injected gas $h_{\mathrm{inj}}$, while in the species continuity equation, the partial densities rates $\rho_{\text {inj } i}$ are calculated by multiplying $\rho_{\text {inj }}$ by mass fractions of the components of the injected gas (hydrogen in this case). The model is capable of simulating the detailed chemistry and $\omega_{i}$ is calculated as a sum of production and depletion of the $i$ th species in all reactions:

$$
\omega_{i}=\frac{d \rho_{i}}{d t}=W_{i}\left[\sum_{k=1}^{m} \pm \nu_{i, k} \alpha\left(k_{k, \text { for }} \prod_{j} C_{j}^{v_{j k}}-k_{k, \text { back }} \prod_{j} C_{j}^{v_{j k}}\right)\right] .
$$

In this paper, a simple single-reaction mechanism is used. The chemical reaction sources are integrated by the quasi-equilibrium solver Chemeq2 [16]. Combustion of hydrogen is described by one reversible reaction:

$$
2 \mathrm{H}_{2}+\mathrm{O}_{2} \Leftrightarrow 2 \mathrm{H}_{2} \mathrm{O}
$$

The forward reaction rate is calculated using the classical Arrhenius equation with the reaction rate constants tuned for a reference case:

$$
k=A T^{n} \exp \left(-\frac{E_{a}}{R T}\right),
$$

while the backward reaction rate is calculated from the assumption of local chemical equilibrium. The reference experimental case was a stable rotating detonation in a chamber $10 \mathrm{~cm}$ in diameter with the mean equivalence ratio 
of 0.5 . The constants are: $A=2.0 \cdot 10^{17} ; n=0.0 E_{a}=14,000$ in a unit system with cal, mole, $\mathrm{cm}$, and s. For these input data, the numerical results agree with experimental measurements within reasonable accuracy.

The model was implemented in the in-house code REFLOPS using structured grids. The Euler equations and the grids can be defined in Cartesian or cylindrical coordinates. Several structured grids can be combined together in order to simulate relatively complex geometries. The code uses HLLC (Harten-LaxLeer-contact) solver in conjuction with WAF (Weighted Average Flux) numerical scheme for the advective fluxes. It is a second-order accurate scheme in space and time [17]. The advective terms are integrated with respect to time using the forth-order Runge-Kutta scheme. While the main objective of the work was to investigate the global behavior of the wave, the relatively low order scheme did not introduce a significant error. Additionally, several cases were calculated for finer grids and no significant discrepancies in the results were obtained in comparison with the original one where the average cell size was $0.5 \times 0.5 \mathrm{~mm}$.

\section{RESULTS AND ANALYSIS}

\subsection{Stability of the Rotating Wave}

The simulation started from searching for a set of injection parameters and initial conditions which ensure generation of a stable rotating detonation. It turned out that in 2D simulation, the range of variation of parameters was significantly narrower that in real three-dimensional (3D) conditions where the process had additional degree of freedom. Additional constraint was the time of calculation. In the experiments, the time between ignition and establishing a stable detonation wave in the chamber was of order of milliseconds. It was prohibitively long for the simulation. Therefore, special initial conditions had been worked out in order to quickly generate a stable rotating detonation and then this case was used as an initial condition for subsequent simulations.

If the injection conditions were improper, two cases were possible: the detonation was extinguished or degraded into a large number of small detonation wavelets propagating chaotically and eventually transiting to deflagration. Figure 16 shows the pressure history after ignition of the mixture when a number of detonation waves were created. It happened when the detonable mixture was created too fast behind the leading wave. Such high pressure and temperature conditions create a new detonation wave moving backward. Subsequent collisions of the waves generate additional hot spots and initiate new detonations. The process either continues or is terminated due to the lack of the fuel when the injection is blocked by high pressure in the combustion chamber. 


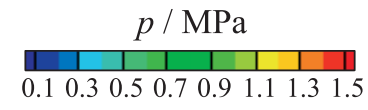

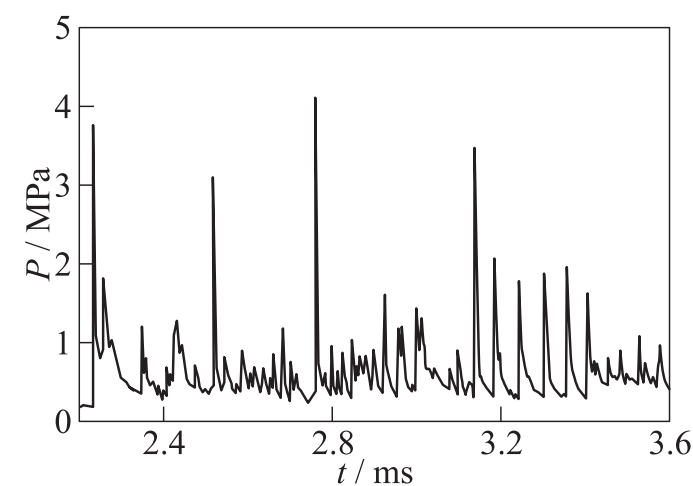

(a)

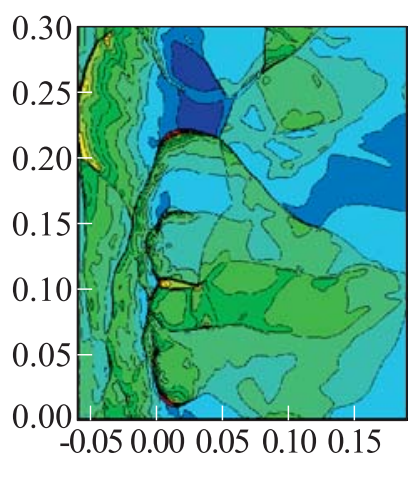

(b)

Figure 16 Pressure-time history of the unstable detonation $(a)$ and the pressure field with several detonation waves (b). (Refer Kindracki et al., p. 570.)

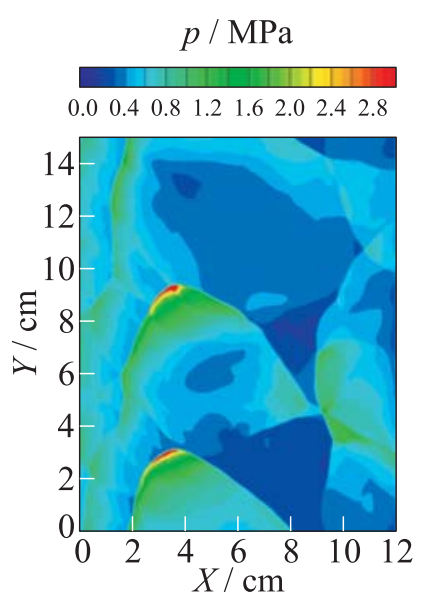

(a)

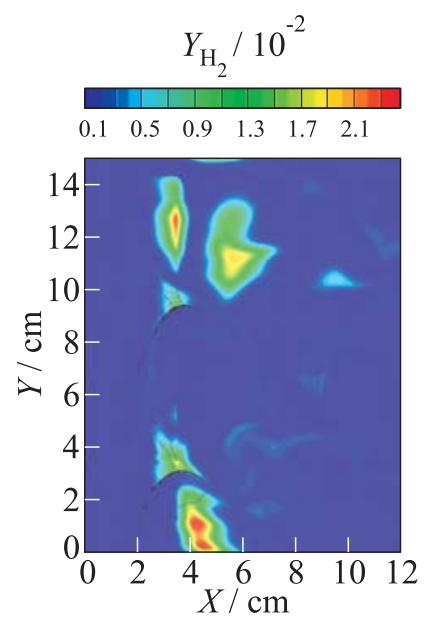

(b)

Figure 17 Instantaneous pressure field (a) and hydrogen mass fraction (color contour) and the pressure (lines) (b) for multihead detonation propagation. (Refer Kindracki et al., p. 570.)

At the intermediate injection conditions between stable and unstable detonation, a metastable propagation of few fronts can be sustained for one or two turns. This multifront detonation is shown in Fig. 17. 
The stable single-head detonation can be achieved when three parameters are adjusted: injection pressure, injection cross-section area, and diameter (circumference) of the detonation chamber.

Figure 18 shows pressure-time histories for stable detonation obtained in the 30-centimeter circumference chamber (mean diameter $9.5 \mathrm{~cm}$ ). The numbering of the numerical sensors and their locations are shown in Fig. 19a. The injection pressure was $p_{\text {inj }}=8$ bar and the mean fuel mass flow rate was about $2.3 \cdot 10^{-2} \mathrm{~kg} / \mathrm{s}$. This mass flow rate corresponded to the overall cross-section area of nozzles equal to $117 \mathrm{~mm}^{2}$ in a 1-centimeter height channel. For two 2D calculations, the height was zero, then the mass flow rate must be recalculated for a proper size of the channel. The same amount of fuel can be also injected through $\Delta=0.39 \mathrm{~mm}$ wide injection slit on the circumference of the chamber. The mean air flow rate was $1.68 \mathrm{~kg} / \mathrm{s}$ that gives the mean stoichiometric coefficient $\Phi=0.48$. At those conditions, the mean tangential component of the detonation velocity (circular velocity) was $V_{c}=1460 \mathrm{~m} / \mathrm{s}$ and the rotation period was $t_{r}=206 \mu$ s.

When the area of the injection nozzle was doubled, the mean value of $\Phi$ increased up to $\Phi=1.07$ and the mean rotation velocity was higher as well. Such a detonation was too fast and the rotation time was shorter than the refilling time necessary for the detonation chamber. Therefore, the size of the detonation chamber was doubled to $19 \mathrm{~cm}$ and stable detonation was obtained at velocity $V_{c}=1806 \mathrm{~m} / \mathrm{s}$ and rotation period $t_{r}=332 \mu \mathrm{s}$. The corresponding pressure history is shown in Fig. 20.

In both cases, the detonation was very stable while there were some fluctuations in the magnitude of pressure peaks similarly to the experiments. This phenomenon is a result of slightly different disposition of the detonation head with respect to the measurement points during the rotations. Very low second and fourth peaks in Fig. 20 are probably a result of first phase of propagation when the wave had not achieved a stationary rotation yet. Further investigations showed that for rich hydrogen-air mixtures, the stable rotating detonation was hardly possible due to high velocity, and larger detonation chambers were probably required for such mixtures.

The next research stage was the investigation of the detonation behavior in larger chambers where the rotation time was much larger than the time required for refilling the channel. For this study, the first set of injection condition was used: $p_{\text {inj }}=8$ bar and $\Delta=0.39 \mathrm{~mm}$. The circumference was 0.6 and $1.2 \mathrm{~m}$. For the first size of the chamber, the detonation was stable. Its tangential velocity was $V_{c}=1426 \mathrm{~m} / \mathrm{s}$ and the rotation period $t_{r}=421 \mu \mathrm{s}$. For the 1.2 -meter circumference, the detonation was stable as well, and the calculated parameters were: tangential velocity $V_{c}=1157 \mathrm{~m} / \mathrm{s}$ and rotation period $t_{r}=1037 \mu \mathrm{s}$. The tangential velocities $V_{c}$ for the circumferences 0.3 and $0.6 \mathrm{~m}$ were almost equal and corresponded to the mean equivalence ratio $\Phi=0.48$. 


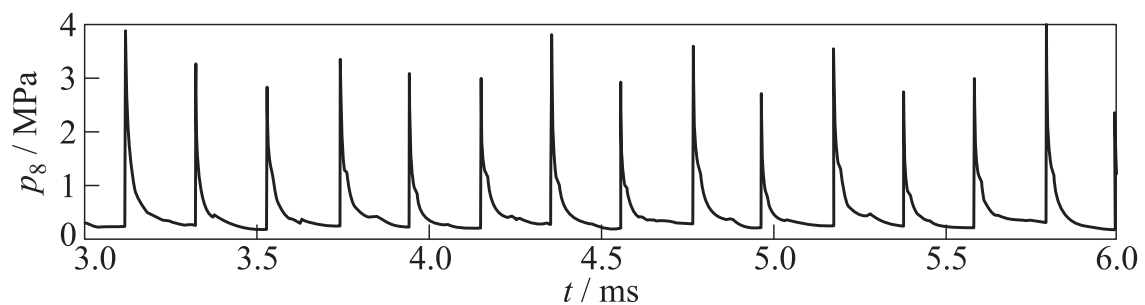

(a)
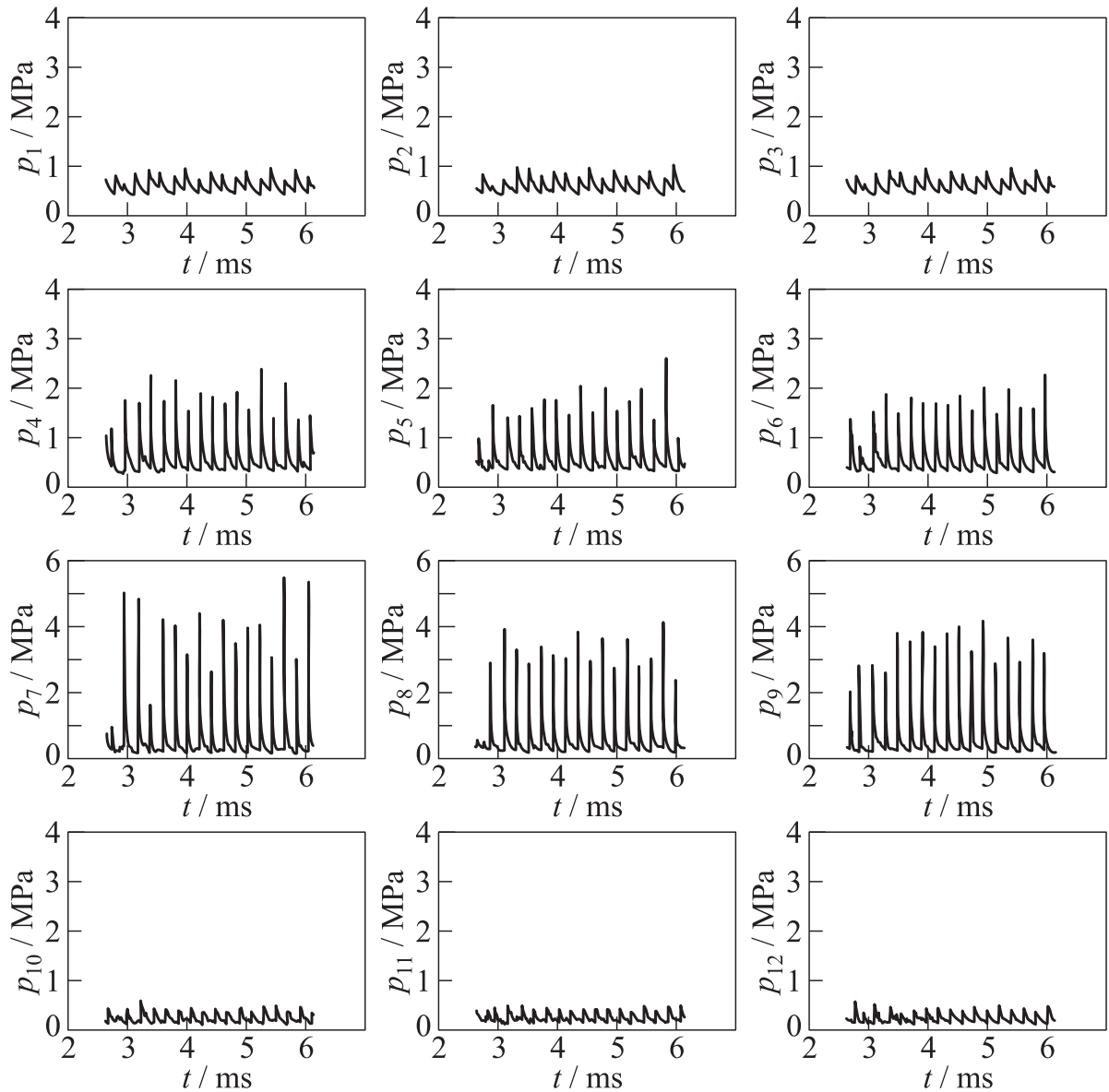

(b)

Figure 18 Predicted pressure histories for rotating detonation in a circular chamber for the circumference of $0.3 \mathrm{~m}$ and mean equivalence ratio of $\Phi=0.48$ (numbering of measurement points is shown in Fig. 19a): $(a)$ is a zoom of the pressure plot $p_{8}$ from $(b)$ 


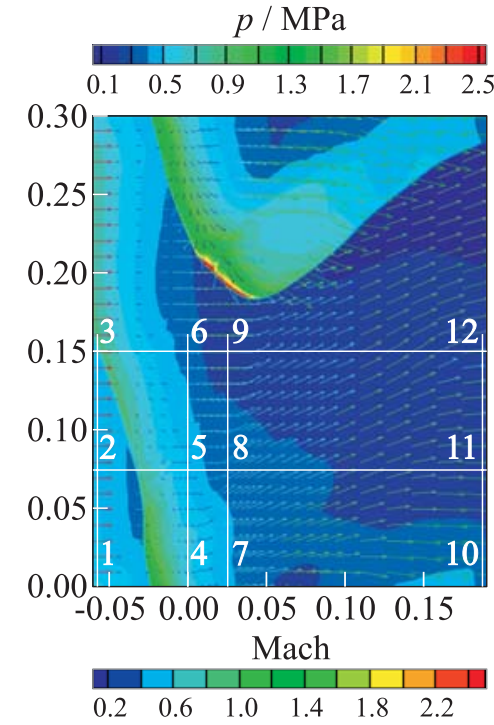

(a)

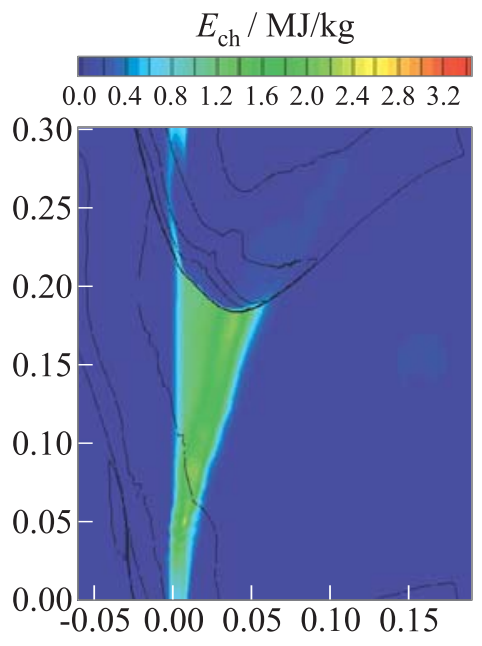

(c)

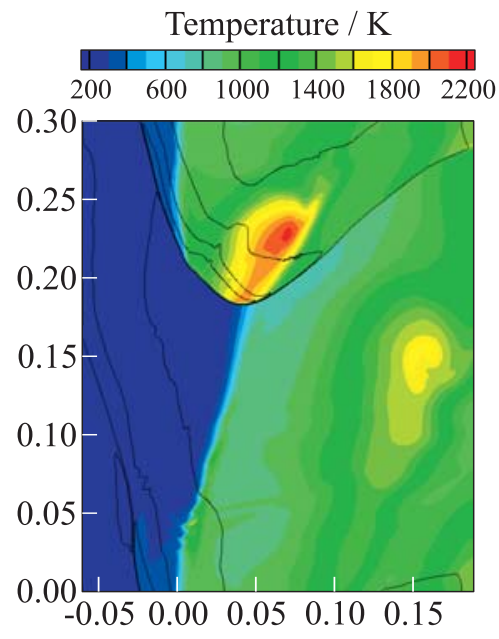

(b)

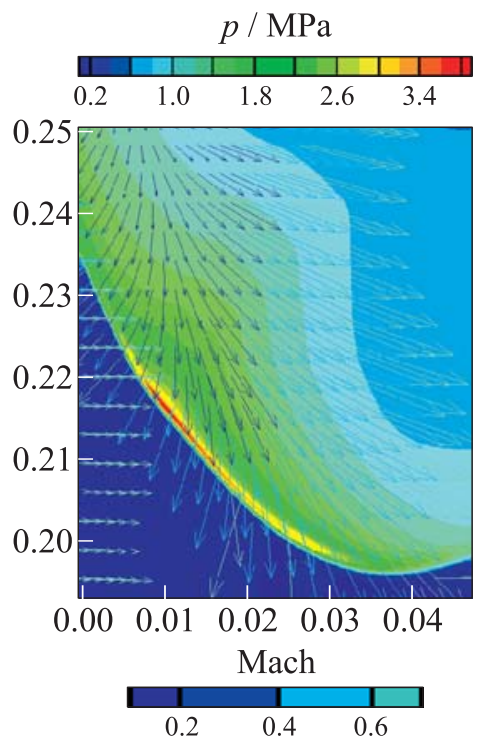

(d)

Figure 19 Rotating detonation flowfield: (a) pressure and velocity (color of the arrows indicates the local Mach number); (b) temperature (with isobars); (c) chemical energy (with isobars) — red color indicates maximum energy for the stoichiometric mixture equal to $3.4 \mathrm{MJ} / \mathrm{kg}$; and $(d)$ detailed pressure and velocity fields at the detonation front. (Refer Kindracki et al., p. 573.) 


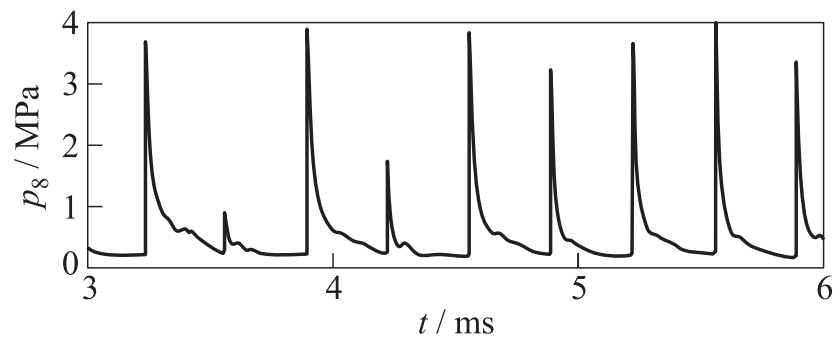

Figure 20 Temporal pressure profiles for rotating detonation in circular chamber for circumference of $0.3 \mathrm{~m}$ and mean equivalence ratio of $\Phi=0.48$ (numbering of the measurement points is shown in Fig. 19 a)

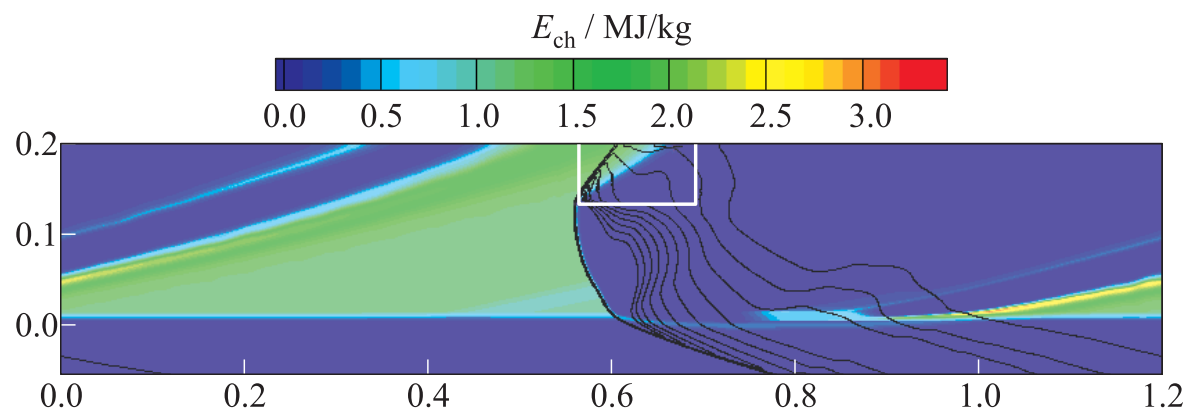

Figure 21 Chemical energy field of mixture $\Phi=0.39$ in the largest chamber (1.2meter circumference), plot is rotated - axial direction is vertical. (Refer Kindracki et al., p. 574.)

The lower velocity for 1.2-meter circumference chamber was caused by two factors. First, the mean equivalence ratio was lower $\Phi=0.39$ as a result of larger amount of colder and denser air flowing above the injector. While the rotation time $t_{r}$ was longer, this extended the time when the cold air was entering the injection region far behind the wave. Then, the mass of air was larger comparing to the mass of fuel. The second reason of reducing the detonation velocity is combustion incompleteness. Simply, the rotation time $t_{r}$ is long enough to allow the mixture flowing through the domain and before it is covered and burnt by the detonation wave. The rectangle in Fig. 21 shows the region where the mixture remains unburnt at the outlet of the chamber. Some amount of the mixture does not react even behind the tail shock wave. It means that even elongating the axial dimension of the chamber would not improve the combustion efficiency when the length of the leading detonation wave is limited by the structure of chemical energy (concentration of fuel and oxygen) deposition. A single rotating 
$p / \mathrm{MPa}$

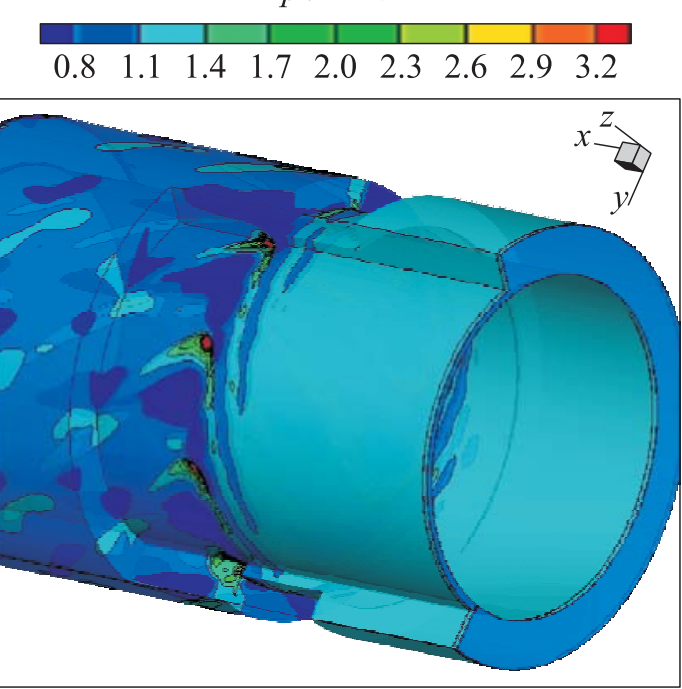

Figure 22 Three-dimensional simulation of a multifront rotating detonation wave. (Refer Kindracki et al., p. 575.)

detonation wave has probably some limit of possible fuel consumption at given injection conditions.

The results presented above were obtained for inviscid model of the gas flow. They showed that a stable single detonation front is possible within the frame of inviscid model. However, in a realistic system, the dissipative processes related to viscosity, heat conductivity, and turbulent mixing introduce additional disturbing factors. The 3D structure of the flow field and the detonation front are also important. The multifront rotating detonations were reported by many experimentalists. Also, preliminary 3D simulations showed that the multiple detonation fronts can be initiated by a complex system of transverse waves in fully $3 \mathrm{D}$ flow. The results of such preliminary simulations are shown in Fig. 22 as an illustration only. However, the problem of the stability of the detonation wave is left for a separate study because of its complexity and necessity of using much more advanced models of the flow.

If the amount of injected fuel (in a 0.3 -meter chamber) is reduced by decreasing pressure by a factor of two, the nozzle is blocked by the backpressure of the detonation for a longer time. At these conditions, the mean equivalence ratio drops to $\Phi \approx 0.2$ which is too low to sustain the detonation.

The tangential velocity $V_{c}$ of the detonation wave is determined by the velocity of the leading edge of the wave. This part of the wave is usually located in the most energetic part of the mixture and the local wave front is parallel to the axis 
Table 1 Tangential velocity and CJ velocity for different sizes of the chamber

\begin{tabular}{cccccccc}
\hline $\begin{array}{c}\text { Circum- } \\
\text { ference, } \\
L, \mathrm{~m}\end{array}$ & $\Phi_{\text {mean }}$ & $\Phi_{\text {loc max }}$ & $V_{c}$ & $\begin{array}{c}V_{\mathrm{CJ}} \\
\left(\Phi_{\text {mean }}\right)\end{array}$ & $\begin{array}{c}\Delta V / V_{\mathrm{CJ}} \\
\left(\Phi_{\text {mean }}\right)\end{array}$ & $\begin{array}{c}V_{\mathrm{CJ}} \\
\left(\Phi_{\text {loc max }}\right)\end{array}$ & $\begin{array}{c}\Delta V / V_{\mathrm{CJ}} \\
\left(\Phi_{\text {loc max }}\right)\end{array}$ \\
\hline 0.3 & 0.48 & 0.706 & 1460 & 1586 & $-8 \%$ & 1801 & $-19 \%$ \\
0.6 & 0.48 & 0.735 & 1426 & 1586 & $-10 \%$ & 1823 & $-22 \%$ \\
$1.2(\mathrm{a})$ & 0.39 & $0.382^{*}$ & 1157 & 1473 & $-21 \%$ & 1472 & $-21 \%$ \\
$1.2(\mathrm{~b})$ & 0.39 & $0.529^{* *}$ & 1157 & 1473 & $-21 \%$ & 1643 & $-30 \%$ \\
\hline
\end{tabular}

* Maximum local value in the front of the detonation wave.

** Maximum local value in the entire flow.

of the chamber and to the mean flow direction of the undisturbed gas. Therefore, one can expect that this velocity should be close to the Chapman-Jouguet (CJ) velocity $V_{\mathrm{CJ}}$ for local thermodynamic conditions. Table 1 shows the relationship between the $V_{c}$ and $V_{\mathrm{CJ}}$ calculated for the mean equivalence ratio and for its maximum local value. The tangential velocity of the wave is lower than the CJ velocity calculated for both, mean and maximum, local values. The discrepancies are caused by rapid expansion of detonation products behind the wave. The gas detonating in a tube can expand only in one, axial direction. Here, the gas expands in two directions. Additionally, the detonation front occupies only a small part of channel cross section. So, for the largest chamber, the discrepancies are larger. However, when the tangential velocity is compared with the CJ velocity for the maximum local equivalence ratio at the detonation front (position $1.2(\mathrm{a})$ ), the relative change is almost the same as for smaller chambers. The results obtained for two cases with $\Phi=0.48$ in small chambers agree well with measurements; however, the direct comparison is difficult because of significant scatter and unsteadiness in the measured velocity.

\subsection{Detonation Structure}

The predicted flow fields are shown in Fig. 19. The wave propagates approximately between injection line which is located along the circumference (vertical direction) at $x=0$ and the line of contact surface between combustion products and fresh mixture. The leading part of the front is created in the region of the highest chemical energy which is also a region of the highest local equivalence ratio. The structure of the field of available energy is obviously strictly connected with the field of hydrogen concentration. In turn, this is a result of the ratio between the local pressure in the chamber and injection pressure, and local air velocity in the vicinity to the injection points. Immediately behind the wave, no fuel is injected due to high pressure in the chamber. Later, in the region where the pressure drops below the injection pressure, the gas velocity is low and more 


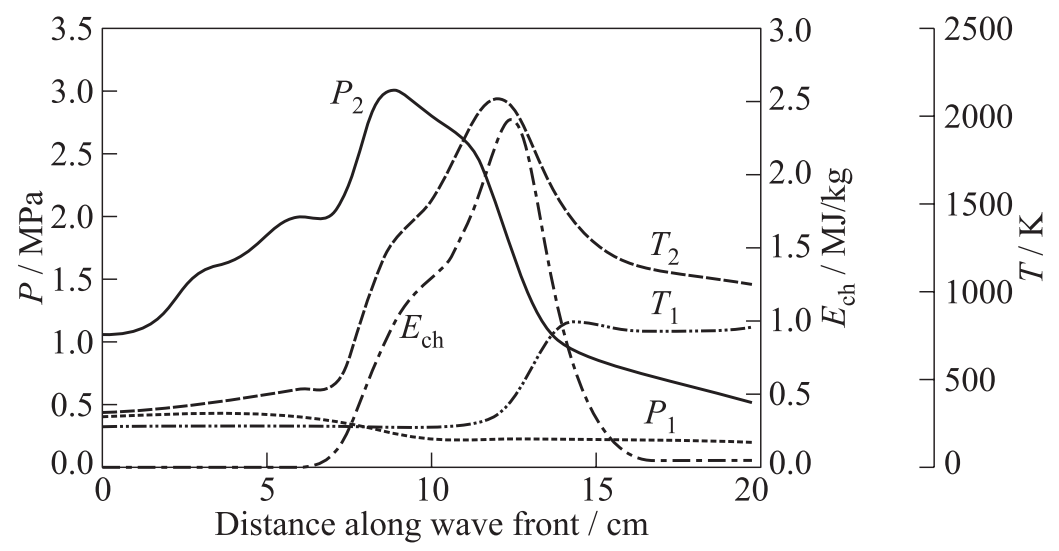

Figure 23 Gas parameters along the detonation front

fuel is injected with respect to the air; therefore, a richer mixture is created. At a long distance from the leading wave front, the axial velocity of air increases and the mixture becomes leaner because of higher local air mass flux. Next, the high-energy richer mixture is advected by gas of higher velocity toward the outlet. Finally, the gas with maximal chemical energy is shaped as an inclined line (Fig. 19c).

Because the wave propagates in the mixture which is not uniform, the parameters behind the wave also vary along the front (Fig. 23). The main factor explaining the structure is available chemical energy which maximum is very close to the mentioned contact line. The pressure $P_{1}$ ahead of the wave is almost constant. The temperature of unshocked gas $T_{1}$ is also uniform except for the contact line between fresh mixture and combustion products which are heated to about $800 \mathrm{~K}$.

The maximum temperature behind the wave front $T_{2}$ is located very close to the maximum of the chemical energy, while the maximum pressure $P_{2}$ is shifted towards the lean mixture (lower energy region). Simple simulation of the detonation propagation in stratified mixture showed that this is the result of an overdriven detonation in the mixture which is less energetic. In a more energetic region, the detonation propagates faster and creates a tail wave in the less energetic mixture. This wave constitutes an overdriven detonation which is characterized by higher pressure and lower temperature.

This leads to the pressure gradient behind the wave which becomes almost axial ( $x$ direction) at some distance from the front. The gradients change the direction of gas motion that is easily visible in Fig. $19 d$. As a result, the mean outlet flow from the chamber is almost axial; however, one can expect stronger 
rotation of the gas caused by the rotating detonation. The almost-axial motion of the outlet gas was also observed in experiments.

Figure 19 shows also that the gas quickly expands behind the wave. Therefore, the static pressure and temperature is low but velocity increases significantly. The temperature does not exceed $1600 \mathrm{~K}$ that is confirmed by the experiments as well. The simulations indicate that a great part of the fuel is burnt at very low temperature of $1500 \mathrm{~K}$. In such conditions, one can expect a reduced production of NOx. However, simulations with detailed chemistry are necessary to prove this implication.

A very important subject for the analysis is the relationship between the local undisturbed gas motion and the wave which is oblique with respect to the vector of gas velocity for leaner part of the mixture. However, the part of the wave which propagates in the mixture of the maximum chemical energy is almost parallel to the direction of the flow. Analytical solution for the oblique detonation wave indicates that the wave is possible only for certain angles between the wave front and velocity vector of unburnt gas. Obviously, the parameters of the gas behind the detonation wave depend on this angle and relative velocity of the gas with respect to the wave. Due to the paper length limitations, these results will be published in the forthcoming papers. Anyway, this theory can help understanding the wave shape and can probably explain why the detonation wave is not created in the region, where a detonatable mixture still exists, like in the flow region in the red rectangle (see Fig. 21).

\subsection{Global Parameters}

From the practical point of view, the most important are the mean total gas parameters at the chamber exit. Figure 24 shows the predicted time histories of total gas parameters and velocity at the inlet and outlet of the chamber. The total pressure in the combustion chamber drops because of the presence of shock waves; however, this disadvantage is compensated by fast and efficient combustion process. Even for a relatively rich mixture, the total temperature is about $1800 \mathrm{~K}$ which is acceptable level for the first stages of modern gas turbine without using a complicated system of air dilution. The mean velocity at the chamber outlet is a factor of 3 higher than that at the inlet. This can be implemented for simplifying the inlet vane design or nozzle design for the rocket engine.

\section{SUMMARY}

The experiments presented here clearly showed a possibility of the continuous propagation of rotating detonation in different cylindrical chambers in a wide 


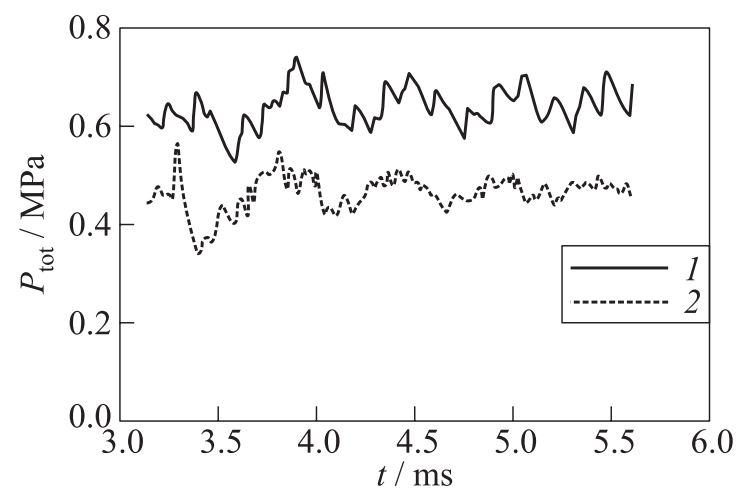

(a)

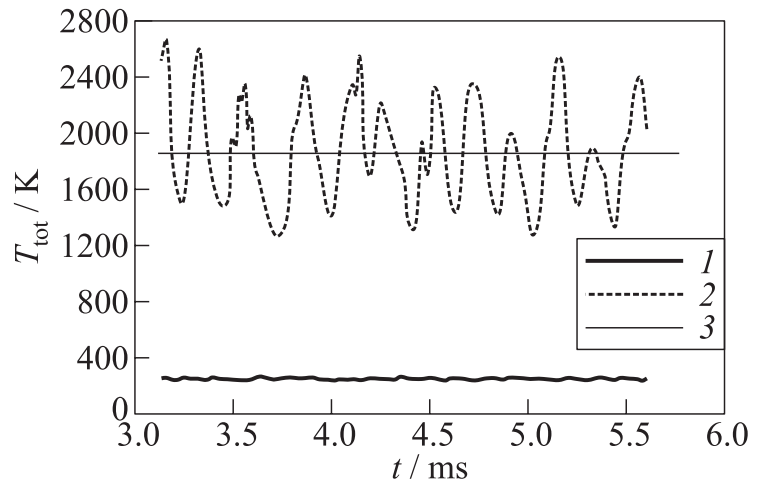

(b)

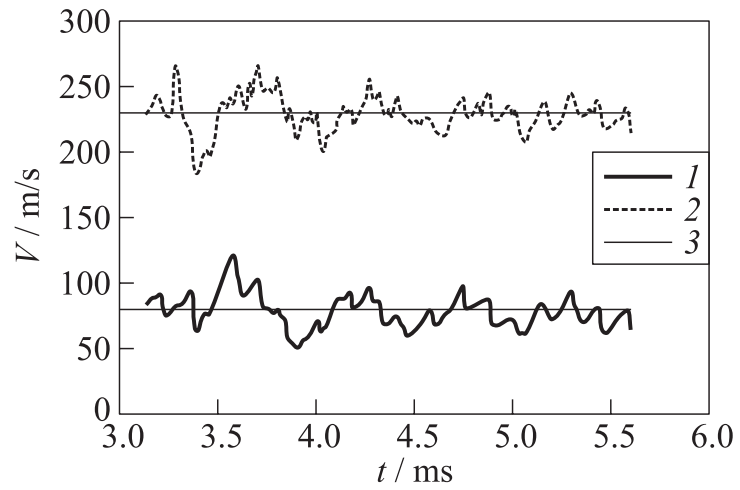

(c)

Figure 24 Mean total pressures $P_{\text {tot }}(a)$, temperatures $T_{\text {tot }}(b)$, and velocities $(c)$ at inlet (1) and outlet (2) of the chamber; 3 - average 
range of fuel-lean hydrogen-air mixtures. The revolution velocity of the detonation wave is a function of thermodynamic parameters and mixture composition. In some cases, instability of the wave was observed both in experiments and simulations. The instability mechanism can be explained by the relationship between the revolution time and refilling and mixing times of the gases in the chamber. The results obtained clearly indicate that the continuous rotating detonation could be applied in jet engines for improvement of their performances. It can be achieved by increasing their efficiency and reducing the weight of the combustion chamber. The next step of the research should be, however, a systematic study of detonation propagating in a mixture of air and jet fuel.

Numerical analysis of the structure of the rotating detonation wave also provided some interesting conclusions. The calculations showed that there exist some range of injection parameters and chamber dimensions where a stable rotating detonation wave could be achieved. It was found that it is much easier to get the rotating detonation for fuel-lean and stoichiometric mixtures than for fuel-rich mixtures. This is because of high detonation velocity of fuel-rich hydrogen-air mixtures. Obviously, for heavier fuels, the situation can be different.

It was also found that the velocity of the wave is lower than the CJ velocity and it mainly depends on the amount of fuel injected. However, the diameter of the chamber cannot be too large because of the possibility of unburned fuel escape between passages of the wave. A possible solution to this problem could be the generation of two detonation heads. Such structures containing two detonation waves were also obtained in these calculations and observed in the experiments.

Analysis of the structure of the rotating detonation showed that the parameters along the wave were not uniform. It was found that the wave was driven by the most energetic mixture but the highest peaks of pressure were created by the overdriven detonation in a less energetic mixture. It produces the axial pressure gradient which accelerates the gas in the axial direction. Finally, the mean flow at the exit from the chamber is almost axial; however, one can expect that the rotation of the wave generates the flow in transverse directions.

The overall feature of the exit gas flow showed that the total pressure slightly decreases because of the presence of shock and detonation waves. Also, the temperatures were observed to be relatively low. Most of the fuel was burnt in the overdriven detonation region at temperatures below $1800 \mathrm{~K}$. This implies an opportunity of reducing NOx emission. Additionally, the mean total temperature was also low, about $1800 \mathrm{~K}$. Such parameters of the exhausted gas allow application of the rotating detonation chamber to modern turbine engines without air dilution. Obviously, it demands further detailed analysis of the rotating wave applications for engines and gas turbine systems. Other fuels must be tested as well. 


\section{ACKNOWLEDGMENTS}

This work was supported by Polish Ministry of Science and Higher Education, project No. 4 T12D 02429.

\section{REFERENCES}

1. Voitsekhovskii, B. V. 1959. Stationary detonation. Dokl. Akad. Nauk SSSR 129(6).

2. Voitsekhovskii, B. V., V.V. Mitrofanov, and M. E. Topchiyan 1963. Structure of the detonation front in gases. Novosibirsk: SO AN SSSR.

3. Wolanski, P., T. Fujiwara, and Mitsubishi. 2004. Patent application for RDE.

4. Bykovskii, F. A. and E. F. Vedernikov. 2003. Continuous detonation of a subsonic flow of a propellant. Combust. Explosion Shock Waves 39(3):323-34.

5. Bykovskii, F. A., S. A. Zhdan, and E. F. Vedernikov. 2005. Continuous spin detonation in annular combustors. Combust. Explosion Shock Waves 41(4):449-59.

6. Bykovskii F. A., S. A. Zhdan, and E. F. Vedernikov. 2006. Continuous spin detonation in fuel-air mixtures. Combust. Explosion Shock Waves 42(4):463-71.

7. Bykovskii, F., S. Zhdan, and E. Vedernikov. 2008. Continuous spin detonation of hydrogen-oxygen mixtures. 1. Annular cylindrical combustors. Combust. Explosion Shock Waves 44(2):150-62.

8. Hishida, M., T. Fujiwara, and P. Wolanski. 2009. Fundamentals of rotating detonations. Shock Waves 19(1):1-10.

9. Davidenko, D. M., I. Gökalp, and A. N. Kudryavtsev. 2007. Numerical simulations of the continuos rotating hydrogen-oxygen detonation with a detailed chemical mechanism. West-East High Speed Flow Field Conference. Moscow, Russia.

10. Davidenko, D. M., I. Gökalp, and A. N. Kudryavtsev. 2008. Numerical study of the continuous detonation wave rocket engine. 15th AIAA International Space Planes and Hypersonic Systems and Technologies Conference.

11. Yi, T.-H., J. Lou, P. Wolanski, et al. 2009. Propulsive performance study of rotational detonation engine. 22nd Colloquium (International) on the Dynamics of Explosions and Reactive Systems. Minsk, Belarus.

12. Wolanski, P., J. Kindracki, and T. Fujiwara. 2005. An experimental study of rotating detonation engine. 20th Colloquium (International) on the Dynamics of Explosions and Reactive Systems. Montreal, Canada.

13. Wolanski, P., J. Kindracki, and T. Fujiwara. 2006. An experimental study of small rotating detonation engine. In: Pulsed and continuous detonation. Eds. G. Roy, S. Frolov, and J. Sinibaldi. Moscow: TORUS PRESS. 332-38.

14. Kobiera, A., M. Folusiak, K. Swiderski, et al. 2009. Three-dimensional modeling of the rotating detonation engine. 22nd Colloquium (International) on the Dynamics of Explosions and Reactive Systems. Minsk, Belarus. 
15. Yi, T.-H., C. Turangan, T. Liu, et al. 2007. Spinning detonation wave in an annular chamber. 20th Symposium (International) on Combustion Processes. Pultusk, Poland.

16. Mott, D. R., and E.S. Oran. 2001. Chemeq2: A solver for the stiff ordinary differential equations of chemical kinetics. NRL/MR/6400-01-8553.

17. Toro, E.E. 1999. Riemann solvers and numerical methods for fluid dynamics. Berlin: Springer. 